Original Research Article

\title{
Electricity Generation through Human Power via Piezoelectric Effect
}

Rana, Ankit Singh ${ }^{1}$ and Srivastava, Sumita ${ }^{2}$

${ }^{1}$ Department of Physics, Pt. L. M. S. Governmemt Post Graduate College, Rishikesh

${ }^{2}$ (Autonomous College) Dehradun -249201, India

Corresponding Author: ranaankitsingh516@gmail.com; sumita_uki1@,rediffmail.com

\section{A R T I C L E I N F O}

Received: 2 July 2018 | Accepted: 02 November 2018 | Published Online: 31 December 2018

DOI: $10.31786 / 09756272.18 .9 .2 .204$

EOI: $10.11208 /$ essence.18.9.2.204

Article is an Open Access Publication.

This work is licensed under Attribution-Non Commercial 4.0 International

(https://creativecommons.org/licenses/by/4.0/)

(CThe Authors (2018). Publishing Rights @ MANU_ICMANU \& ESSENCE_IJERC.

\section{A B S T R A C T}

The piezoelectric effect is the phenomenon of conversion of mechanical energy into electrical energy. In the circular piezoelectric transducers the variation of output voltage obtained was measured with respect to changes in various parameters to get maximum output voltage. The whole experimental work was divided into two parts - without capacitor (AC output) and with capacitor (DC output). Studies were made to observe effects of different weights, surface of various thickness and rigidity used below the piezoelectric material as base. This resulted in increased output ac voltage with increase in weight, elasticity and thickness of the material. In next experiment, piezoelectric crystal was applied on the sole of the shoes to see the variation in voltage generated with the number of taps taken at one place, number of steps taken while walking and the rate of change of taps taken. The voltage generated in case of walking was more in comparison to tapping of feet standing at one place. The output voltage also increases with increased rate of tapping the feet. In addition to this, a "Piezoelectric Chakla (Rotimaker)" was designed by fixing a layer of these piezoelectric disc elements. Whenever the wooden roller is rolled above this Chakla or Rotimaker there is generation of voltage sufficient to charge a mobile or a battery. These little steps when added together can save a sufficient amount of energy in our society.

\section{K E Y W O R D S}

Piezoelectricity | Human power | Piezoelectric shoes | Rotimaker

\section{I T A T I O N}

Rana, Ankit Singh and Srivastava, Sumita (2018): Electricity Generation through Human Power via Piezoelectric Effect. ESSENCE Int. J. Env. Rehab. Conserv. IX (1): 23-27. 


\section{Introduction}

The piezoelectric energy or the pressure energy is the phenomenon discovered by Pierre \& Jacques Curie in 1880, as they were the first to determine the generation of electricity (surface charges) through the well prepared quartz crystals on application of mechanical pressure. In 1881 the term "piezoelectricity" was first suggested by W. Hankel, and the converse effect was mathematically deduced by Gabriel Lipmann from fundamental thermodynamic principles according to which on application of an external voltage across a piezoelectric material, it undergoes mechanical distortion as a result. Crystals like quartz, tourmaline and Rochelle salt exhibit such piezoelectric properties. The traditional single crystal materials suffer from various disadvantages limiting their use to some extent. The limitations include weak piezoelectric effect, low mechanical strength, sensitivity to moisture and their operated temperature range is often limited. Busch and Scherrer in 1935 discovered piezoelectric effect in Potassium Dihydrogen Phosphate (KDP) and its isomorph. The first major family of the piezoelectrics and ferroelectrics was this KDP family.

Unusual dielectric properties like abnormally high dielectric constant were found in $\mathrm{BaTiO} 3$ (Barium titanate) during 1940 to 1943 independently by Wainer and Salmon, Ojawa, and Wul and Golman. After this some compositional modifications led to improvement in temperature stability and high voltage output in $\mathrm{BaTiO}_{3}$ ceramics. $\mathrm{BaTiO}_{3}$ ceramics usually have high dielectric constant due to their ferroelectric (permanent internal dipole moment) nature thus giving rise to new class of ferroelectrics with the $\mathrm{ABO}_{3}$ perovskite structure. In 1950s, Jaffe and his co-workers discovered the PZT system that was capable of holding strong piezoelectric effects. PZT compositions near the morph trophic phase boundary (MPB) between the rhombohedral and tetragonal phase's exhibits the maximum piezoelectric response making it dominant piezoelectric ceramic for a variety of applications. Nowadays Lead Zirconium Titanate (PZT) and Barium Titanate $\left(\mathrm{BaTiO}_{3}\right)$ having a great advantage of large and stable piezoelectric effect, high strength and ease of fabrication in the various fields of piezoelectric applications like actuators, sensors, and smart structures dominating the markets of the world today.

In present investigations, piezoelectric materials have been used to design certain new devices to produce electricity through human power. The details are being described here.

\section{Experimental Details}

"The piezoelectric effect is the phenomenon of conversion of pressure (mechanical) energy into electrical energy." Here in present case, the use of circular piezoelectric transducers have been used to measure the variation of output voltage obtained with respect to changes in various parameters and how by utilizing these changes one can gain the maximum output voltage. The whole experiment work was divided into two parts - without capacitor (AC output) and with capacitor (DC output). So we proceeded as follows-

- Firstly, the effects of the amount of pressure applied upon the circular piezodisc in the form of different weights were observed.

- Secondly, the effect of surface used below the piezoelectric material as base and its effect on the amount of voltage generated as output was observed. So different surfaces of various thickness and rigidity below this piezoelectric element were applied. These experimental set-ups are shown in Fig: 1a and 1b.

Now proceeding with the second half, a bridge rectifier (with capacitor) was made to obtain a DC output voltage. In this case, piezoelectric shoe was prepared with piezoelectric disc elements. These piezoelectric disc elements were fixed on the sole of the shoe from inside to measure the variation on output voltage generated with the number of 


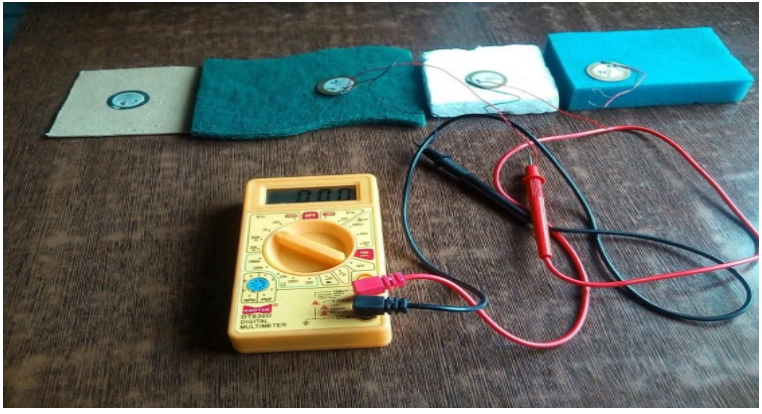

Fig. 1 (a): Experimental set up for measuring piezoelectric voltage to see variation through base.

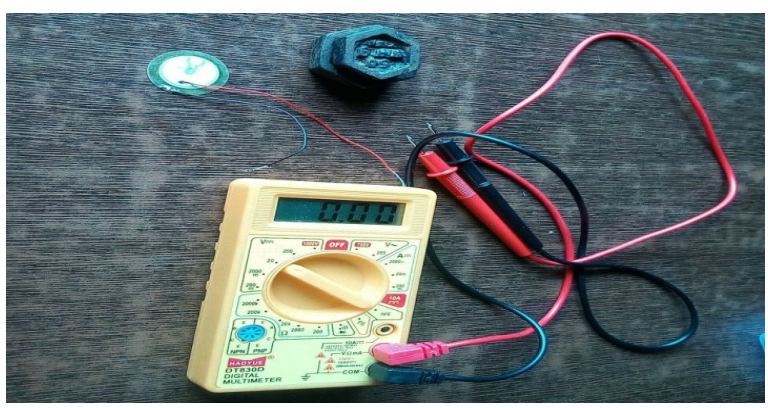

Fig. 1(b): Experimental set up for measuring piezoelectric voltage to see variation through weight

taps taken at one place, number of steps taken while walking and the rate of change of taps taken. The circuit design of piezoelectric shoe is presented in Figs 2a, $2 \mathrm{~b}$.

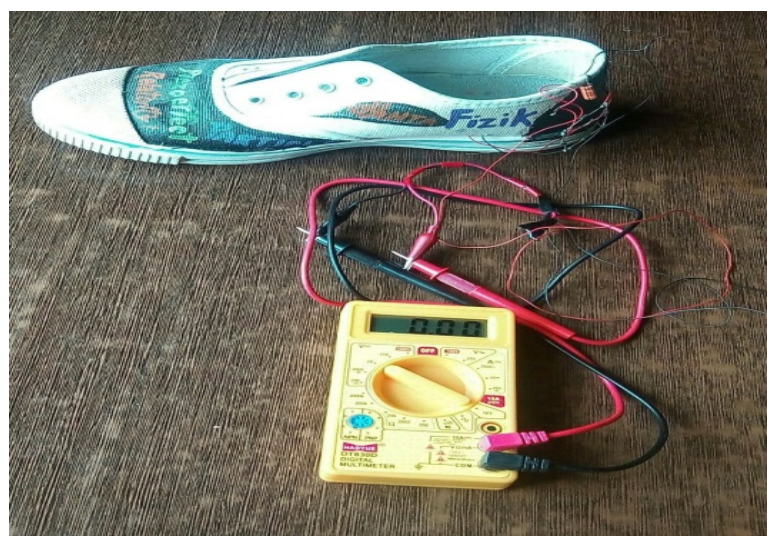

Fig. 2(a): Piezoelectric shoes.

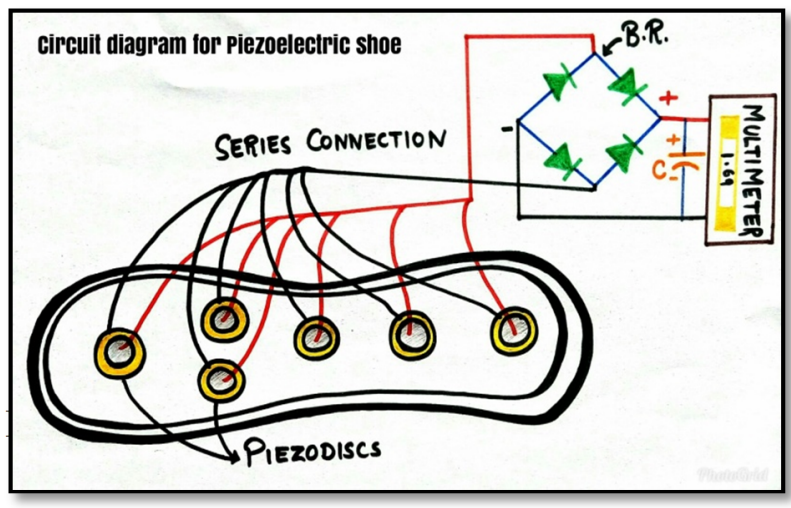

Fig. 2(b): Piezoelectric circuit (i). With increased amount of pressure (here different weights), output voltage generated also increases. The effect is given in Fig 3.

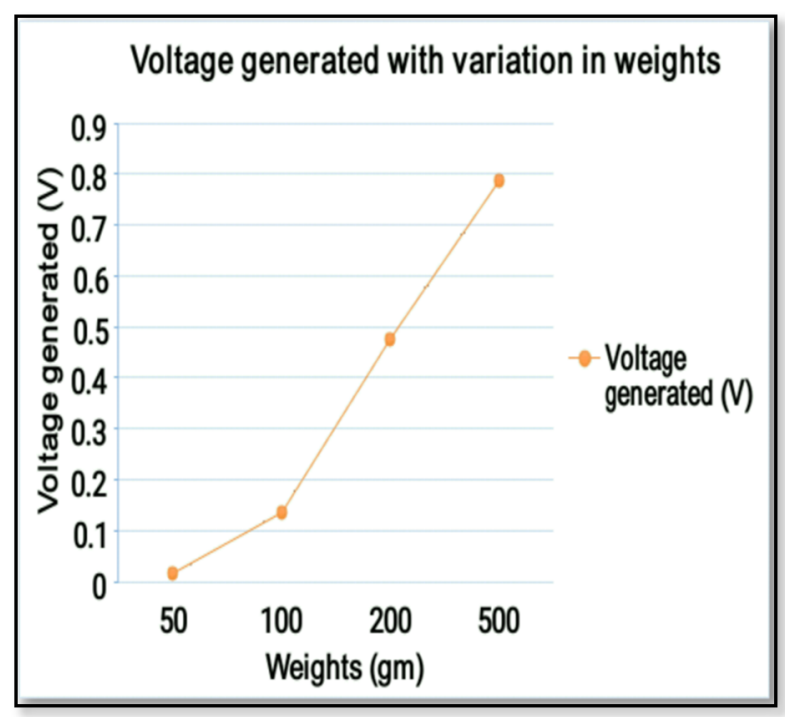

Fig. 3: Variation in output voltage with applied.

(ii). Increasing elasticity and thickness of the material used as base below the piezoelectric material output increases. The variation is represented in Fig 4.

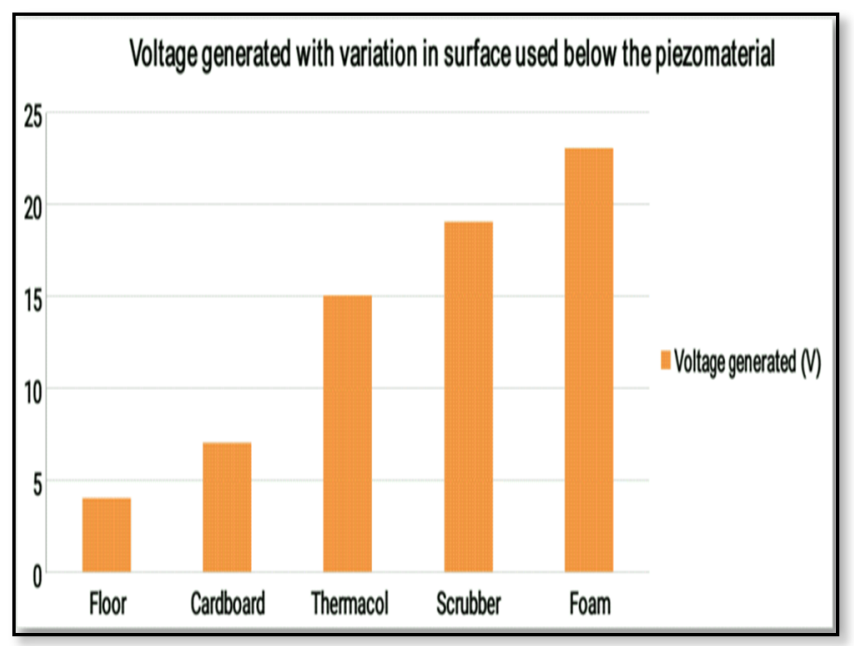

Fig. 4: Variation in output voltage with different base material.

(iii). By increasing the number of taps taken at one place, the amount of output voltage generated also increases.

(iv). With every step taken while walking, the output voltage generated also increases.

(v). Comparing the above two, it is observed that the output generated in case of walking is more in 
comparison to tapping of feet standing at one place.

(vi). The output voltage also increases with increased rate of tapping the feet. This variation is shown in Fig 5.

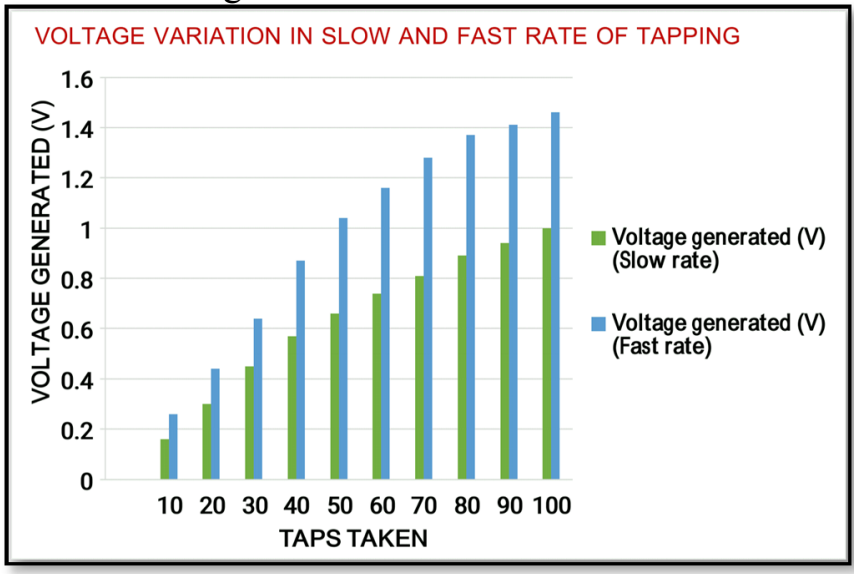

Fig. 5: Variation in output voltage with rate of tapping.

(vii) Using capacitors of small capacitance irregular output is obtained whereas the use of capacitors of higher capacitance yields a much stable output. This effect is presented in Fig 6a, 6b.

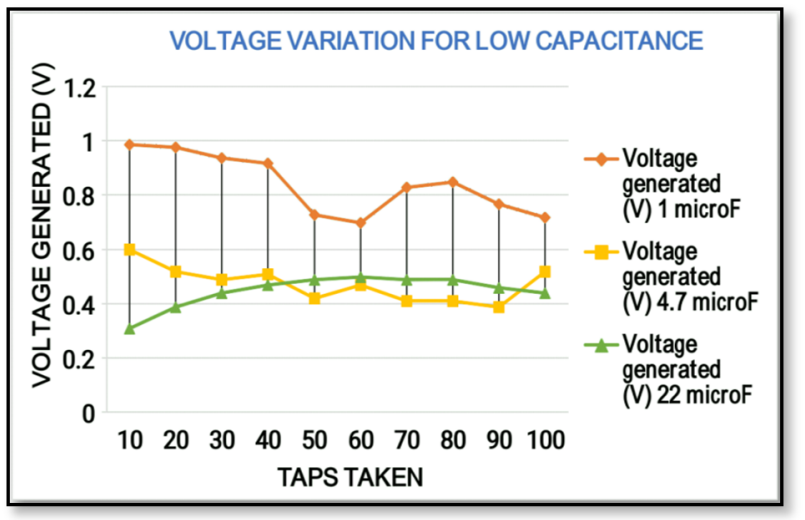

Fig. 6a: Variation in output voltage with application of low capacitance.

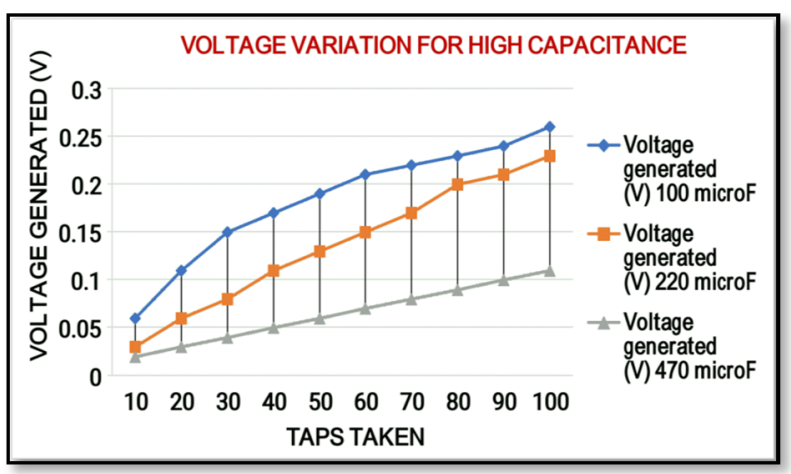

Fig. 6b: Variation in output voltage with application of high capacitance

\section{Application}

As it is well known fact that in India the most handy tool in use for domestic purpose is the Chakla (Rotimaker) and it can be easily utilized to generate a sufficient amount of energy using the oscillatory pressure made on its surface during preparation of Chapati.

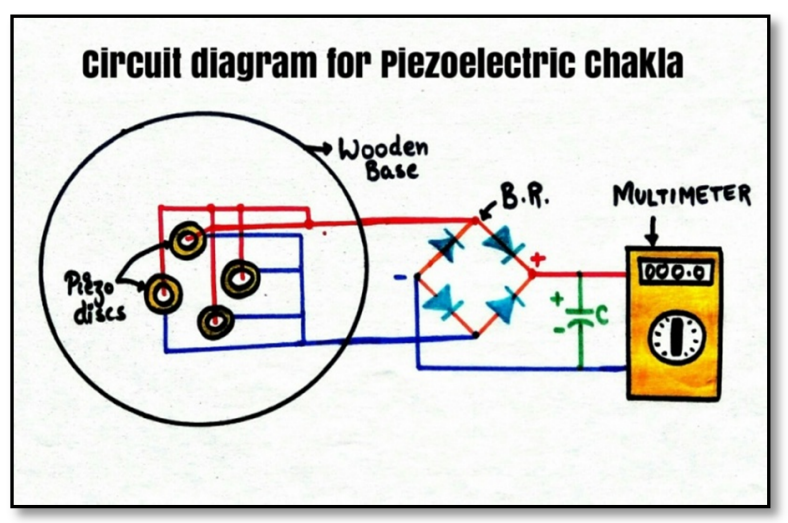

Fig. $7 a$

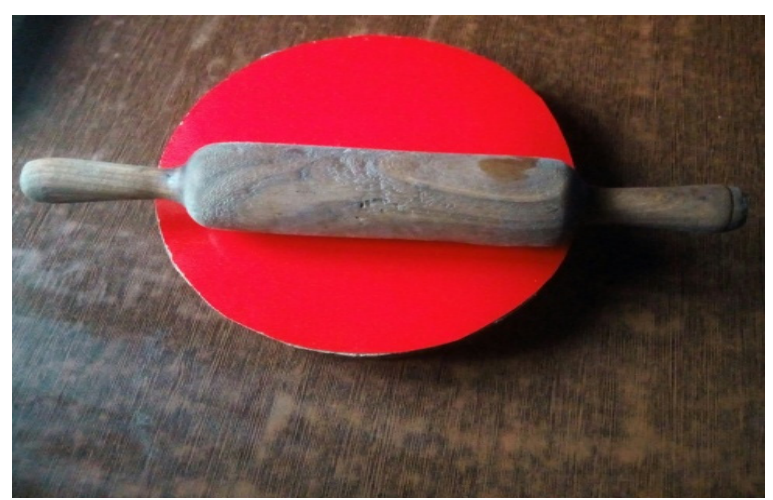

Fig. 7b: The circuit of Rotimaker to produce piezoelectricity

\section{Conclusions}

Imagine that a whole day work may yield a free electricity bill of half of the day or a sufficient amount of energy that can be stored for future use. It seems to be fantasy but with new ideas incorporated in this context, a new level of energy conservation can be visualized. This application is shown in Fig 7a, 7b. Piezoelectric effect is based on basic rule of application of pressure in order to obtain a voltage as output but with the condition that an oscillatory motion is needed to be applied to the piezo materials. Using the above results one can use piezoelectric materials in a much 
innovative way like the one made in the present investigation by applying these piezoelectric transducers to construct a piezoelectric shoes and Chakla (Rotimaker). It shows that the use of piezomaterials should not be restricted only to industrial use but can be made applicable to innovative creation as well as they can be a part of daily life activities. Further study on this subject is open to anybody as these wonder materials can play a vital role in conservation of energy in the upcoming future and may surely achieve the title of next generation eco source.

\section{Acknowledgements}

Authors are grateful to Dr. N. P. Maheshwari, Principal, Govt. P.G. College Rishikesh for his encouragement and Dr. D. P. Bhatt, HOD Physics of Govt. P.G. College, Rishikesh for helpful discussions.

\section{References}

Cady, W. G. (1964): Piezoelectricity: An introduction to the theory and applications of electromechanical phenomena in crystals; Dover Publications: New York, NY, 1-20.

Haertling, G. H. (1999): J Am Ceram Soc. 82, 797-818.

Lippman, G. (1881): Annales de chimie et de physique, 24, 145-178, Theory of Piezoelectric Materials and Their Applications in Civil Engineering- By Antoine Leodoux.

Wersing, W. (2002): In Piezoelectric Materials in Devices ; Setter , N.; Ceramic Laboratory , EPFL Swiss Federal Institute of Technology , Lausanne, Switzerland, 2966. 